\title{
ВАРИАБЕЛЬНОСТЬ РЕФЕРЕНТНОГО ДИАПАЗОНА УРОВНЯ ВИТАМИНА D ПО ДАННЫМ НАЦИОНАЛЬНОГО ОПРОСА ВРАЧЕЙ
}

\author{
Катамадзе Н.Н., Шутова А.С., Фаргиева Х.Р., Поваляева А.А., Пигарова Е.А., Дзеранова Л.К., Глазьева В.С., \\ Беловалова И.М., Дедов И.И. \\ ФГБУ «НМИЦ эндокринологии» Минздрава России, Москва, Россия, \\ Московский государственный университет имени М.В. Ломоносова, России, Москва
}

Связь между дефицитом витамина D и остеопорозом хорошо изучена. Гиповитаминоз витамина D может приводить к воспалительным и аутоиммунным заболеваниям. Однако и обратная ситуация является опасной для здоровья. Для токсичности витамина D характерны такие симптомы, как тошнота, рвота, запор, панкреатит, острая почечная недостаточность, снижение массы тела, обезвоживание, нарушение психического состояния, когнитивные расстройства и развитие жизнеугрожающих состояний.

ЦЕЛЬ: заключалась в оценке вариабельности верхних и нижних границ референтного диапазона 25(OH)D.

МЕТОДЫ: мы провели национальный опрос среди врачей, специализирующихся в различных областях клинической медицины. 80\% из них - эндокринологи.

РЕЗУЛЬтАТЫ: в ходе нашего опроса было установлено, что из 704 врачей 77 (10,9\%) и 45 (6,4\%) рассматривают в качестве нижних границ референтного интервала 25 нмоль/л и 50 нмоль/л соответственно. При этом 111 (15,8\%), 115 (16,3\%) и 36 (5,1\%) врачей рассматривали 375 нмоль/л, 150 нмоль/л и 75 нмоль/л в качестве верхних границ референтного интервала соответственно.

Выводы: в настоящее время вопрос об имплементации единых референтных значений 25(OH)D в сыворотке крови остается нерешенным, несмотря на утверждение специализированных клинических рекомендаций ведущих профессиональных сообществ. Повышение уровня информированности медицинских работников в этой области, а также расширение знаний о характеристиках кальций-фосфорного обмена поможет поддерживать адекватные уровни витамина D и избежать гипервитаминоза. B настоящее время врачи при назначении витамина D должны проявлять осторожность во избежание пагубных для здоровья пациентов последствий. 